\title{
Carcinogen Screening
}

National Cancer Institute

\section{Source}

National Cancer Institute. Carcinogen Screening. NCI Thesaurus. Code C15779.

In vitro or in vivo testing to give a quantitative measure of tumor-inducing potency of the substance. 